\title{
Construyendo la imagen de una ciudad segura: la disminución de la violencia visible en Río de Janeiro
}

\author{
Sebastián Saborío
}

Invitado nacional

Escuela de Sociología de la Universidad de Costa Rica, Costa Rica

sebastian.saborio@gmail.com

Recibido: 21 de julio del 2017

Aprobado: 13 de noviembre del 2017

\section{Sebastián Saborío}

Licenciado en Ciencias Políticas y Relaciones Internacionales y Master en Sociología de la

Universidad de Padua, Italia. Doctor en Sociología por la Universidade Federal do Rio de Janeiro y por la Università degli Studi di Urbino. Docente de la Escuela de Sociología de la Universidad de Costa Rica.

\section{Resumen:}

Desde el 2008 en Río de Janeiro se ha puesto en práctica una nueva política de seguridad pública: la pacificación de las favelas. Esta consiste en la tomada de control, por parte de las Unidades de Policía Pacificadora (UPPs), de algunas de las favelas ocupadas por grupos criminales fuertemente armados. El presente artículo demuestra que, contrariamente a lo que afirman las instituciones locales, la pacificación de las favelas no tiene como objetivo principal "llevar la paz" dentro de territorios urbanos caracterizados por altos índices de violencia. Aquí se sostiene que las UPPs hacen parte de una estrategia más amplia de reducción de la visibilidad de la violencia y, en consecuencia, de la fama de Río de Janeiro de ser una ciudad peligrosa.

Palabras clave: imaginario urbano; políticas de seguridad; unidades de policía pacificadora; violencia; Rio de Janeiro.

Creating the Image of a Safe City: Reduction of Visible Violence in Rio de Janeiro

\begin{abstract}
:
Recently, a new policy of public security has been instated in Rio de Janeiro, aiming for the pacification of the "favelas". This policy gives control to the UPP (Pacifying Police Units) of some favelas occupied by heavily armed groups. This article shows that, contrary to what local institutions declare, the pacification processes in the favelas do not actually seek "bringing peace" to these urban territories characterized by a high index of violence. In this article, it is argued that the UPPs are part of a bigger strategy which aims to reduce the visibility of violence, and, as a result, the common perception of Rio de Janeiro as a dangerous city.
\end{abstract}

Keywords: urban imagery; security policies; pacifying police units; violence; Rio de Janeiro. 


\section{Introducción}

n el 2009, luego del veredicto positivo del Comité Olímpico Internacional (COI) y de

la Fédération Internationale de Football Association (FIFA), Río de Janeiro pasó a la historia como la primera ciudad en la que se llevarían a cabo, consecutivamente, los dos eventos deportivos más grandes y prestigiosos a nivel mundial: el Mundial de fútbol del 2014 y los Juegos Olímpicos del 2016. El primero de estos, aunque contó con la participación de doce ciudades, vio a Río de Janeiro como mayor protagonista. De hecho, en el Maracaná, el famoso estadio de la ciudad, se jugaron varios partidos, entre los cuales estuvo la final del campeonato. Por otra parte, las Olimpiadas tuvieron como única sede Río de Janeiro.

Conocida mundialmente por sus elevados niveles de violencia (Huguet \& Szabó de Carvalho, 2008; Monteiro, 2013), Río de Janeiro tuvo que demostrar, a la FIFA y al $\mathrm{COI}$, que podía garantizar la incolumidad de los turistas internacionales. Desde el principio del proceso de selección de las ciudades huéspedes del mundial de fútbol y las Olimpiadas, estas instituciones manifestaron abiertamente su preocupación en relación a las condiciones de seguridad de la ciudad. En particular, la presencia de bandas criminales fuertemente armadas en favelas posicionadas en las cercanías del estadio Maracaná y otros lugares turísticos se presentaba como uno de los mayores obstáculos a la realización de los eventos.

Hospedar los mega eventos deportivos fue, para la ciudad, una forma de sostener y acelerar el proceso de atracción de capitales globales dentro del territorio local. La necesidad de mostrarse capaces de organizar tales eventos tenía, entonces, un valor económico para los gobernantes y sus aliados del sector privado. Por estas razones, las autoridades tuvieron que demostrar que Río de Janeiro, conocida como una de las ciudades más peligrosas del mundo, podía volverse también un lugar seguro para hacer negocios (Saborio, 2013).

Es en este contexto fue que, el 19 diciembre del 2008, la Policía Militar ${ }^{1}$ puso en marcha el proceso de "pacificación" de algunas de las favelas de la ciudad. Sus objetivos oficiales fueron los de recuperar parte de las favelas ocupadas por bandas de narcotraficantes armados, promover relaciones positivas entre la población local y los miembros de las policías y, en general, de prevenir la violencia urbana. Hasta hoy, se cuenta con la presencia estable de "Unidades de Policía Pacificadora" (UPPs) en 264 de las más de 1.000 favelas de la ciudad, la mayoría de las cuales están ubicadas en los entornos de los barrios más valorizados económicamente y donde se llevaron a cabo los mega eventos deportivos arriba mencionados. Entonces, es posible afirmar que la decisión de "levar la paz" dentro de las favelas, coincidió con la voluntad de las fuerzas políticas locales de demostrar a la FIFA y al COI su capacidad de mejorar las condiciones de seguridad en las zonas de la ciudad envueltas en la organización y la implementación de los mega eventos ${ }^{2}$.

1 En Brasil, la Policía Militar se ocupa únicamente de patrullar el territorio, mientras que la Policía Civil hace trabajo de investigación.

2 La figura (Cano \& Ribeiro, 2014) nos muestra como las favelas controladas por parte de las Unidades de Policía Pacificadora (en amarillo) están situadas principalmente alrededor de las áreas más valorizadas de la ciudad (Zona Sur, Zona Norte en los alrededores del estadio Maracaná y Centro).

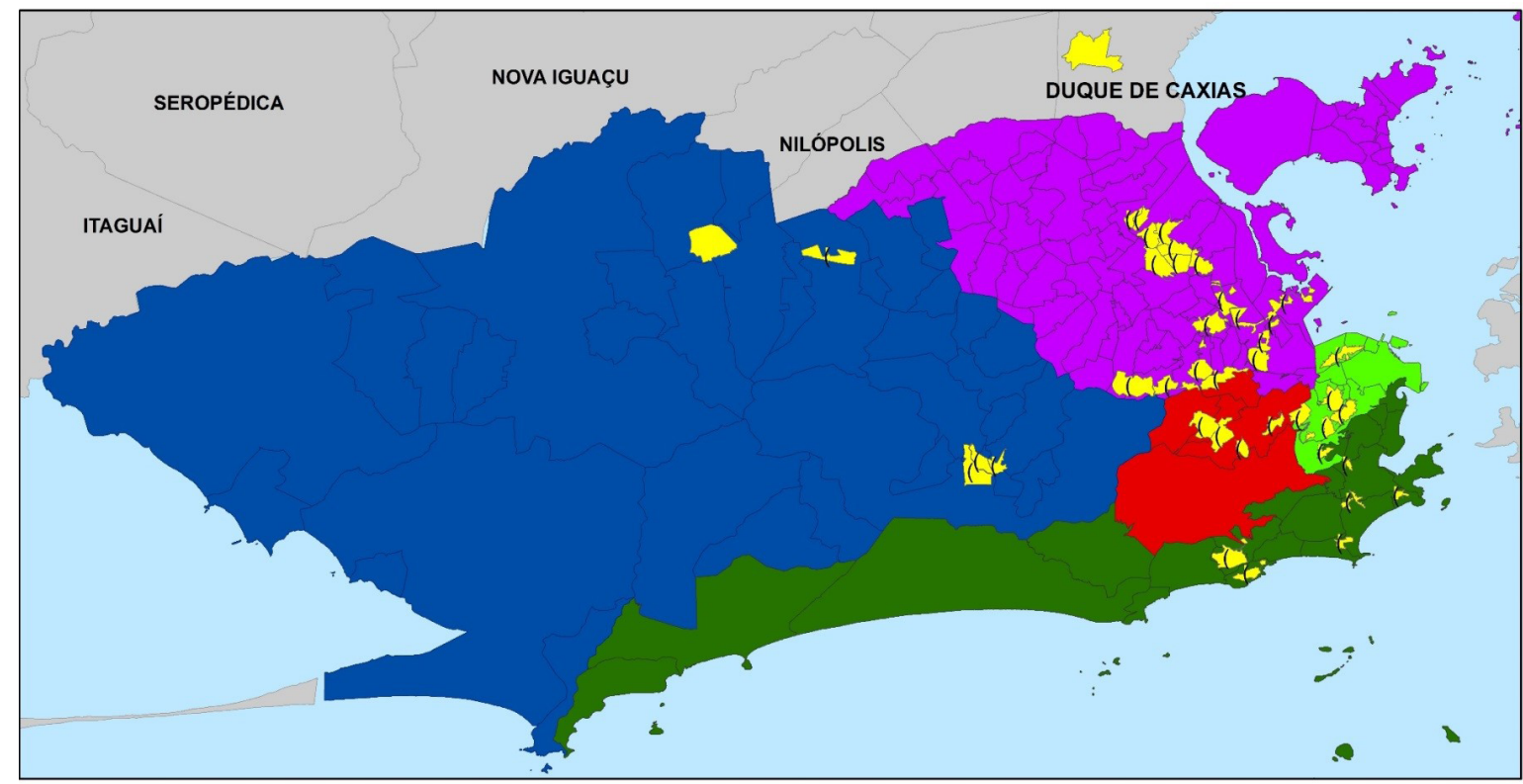

Legenda:
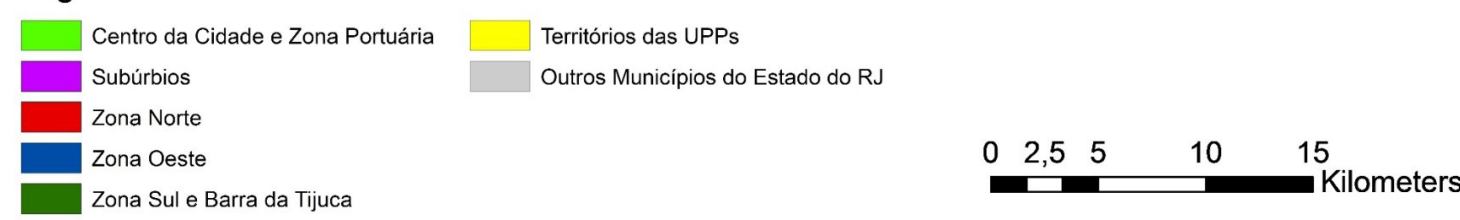
El programa de pacificación ha sido señalado, por parte de las autoridades locales, como el responsable de la fuerte disminución de homicidios que se verificó en la ciudad en años recientes. Sin embargo, como se demostrará en las próximas páginas, las UPPs forman parte de una estrategia más amplia de disminución de la visibilidad de la violencia urbana que, desde los años cincuenta del siglo pasado, fue aumentando hasta el punto de permitir que Río de Janeiro fuera identificada internacionalmente como una "ciudad en guerra" (Pereira Leite, 2012).

\section{La acumulación de la violencia visible}

Para poder entender la situación actual de Río de Janeiro en relación a la violencia, es necesario hacer un excursus histórico a comenzar en los años 1950, época en la que, según Michel Misse (2008), la criminalidad en la ciudad fue transformándose y asumiendo formas más violentas y visibles respecto al pasado. Antes de ese periodo, los crímenes más frecuentes y los que recibian mayor atención en la opinión pública eran, casi exclusivamente, aquellos contra el patrimonio y que "no involucraban el uso de la fuerza o su amenaza" (Misse, 2008, p. 375). A partir de la década de los cincuenta del siglo pasado, en la ciudad se verificó un aumento de los asaltos a mano armada en vía pública y en bancos, así como de robos dentro de las habitaciones privadas. Todos estos crímenes desencadenaron fuertes preocupaciones por parte de la ciudadanía y, en consecuencia, recibieron una amplia atención por parte de la prensa local.

La respuesta que se dio por parte de las autoridades fue la creación de los primeros "escuadrones de la muerte", grupos de policías que tenían la tarea de ajusticiar, de forma extralegal, los sospechosos de cometer este tipo de crímenes. Tal práctica fue el resultado de una idea difusa, todavía hoy en día, en la realidad social brasileña, según la cual "el único criminal bueno es el criminal muerto". Además de no conseguir resolver el problema de la criminalidad, los "escuadrones de la muerte" empezaron lo que Misse (2008) llama la "acumulación social de la violencia"; o sea, una espiral sin fin en la que la violencia de una parte alimenta inexorablemente la violencia de la otra.

Aunque en esos años la visibilidad de la violencia aumentó considerablemente, el índice de homicidios todavía era relativamente bajo respecto al que alcanzaría la ciudad en los años por venir. La tasa de homicidios pasó de 10 de cada 100.000 habitantes, en los años 1950, a 50 de cada 100.000, en los años 1980 (Misse, 2011) y, en 1994, año en el que se tocó el ápice, a 74 homicidios cada 100.000 habitantes (Monteiro, 2013). A medida que la violencia física creció de "frecuencia, intensidad y, principalmente, visibilidad"3 (Machado da Silva, Pereira Leite \& Fridman, 2005, p. 11), la ciudad pasó a ser percibida no sólo como violenta, sino que, incluso, como fragmentada y en guerra.

El aumento de los homicidios coincide con la territorialización del narcomenudeo en las favelas, las guerras territoriales entre bandas criminales y la respuesta fuertemente represiva por parte de la policía (Saborio, 2016). El nivel de la violencia fue tal que en la ciudad se dieron más muertes que en algunos contextos de guerra declarada. Por ejemplo, en los mismos años en los que se llevaban a cabo, los conflictos de "Angola (1998-2000), Sierra León (1991-1999), Yugoslavia (1998-2000), Afganistán

3 Todas las citas de textos en idiomas diferentes al español han sido traducidas literalmente por parte del autor.

\section{$4 \quad$ Los residentes de Río de Janeiro. \\ $5 \quad$ Robos "en masa" perpetrados por} parte de jóvenes provenientes de las favelas, principalmente en las playas de la ciudad y otras localidades turísticas y barrios de las clases medias y altas. (1991-1999) e Israel (1991-1999)" (Ramos \& Lemgruber, 2004, p. 1) en Río de Janeiro murieron más personas de heridas por armas de fuego.

Los homicidios no son el único factor que favoreció la creación de la idea de que, como sostiene Penglase (2014, p. 13), la violencia urbana en Río de Janeiro sea la consecuencia de una "guerra que no es una guerra". Para Pereira Leite (2012), el uso creciente, por parte de la opinión pública, de la "metáfora de la guerra" se debe, de hecho, a una ulterior transformación de la violencia que se verificó en la ciudad a partir de los años de 1980. En esos años, nuevas y más visibles formas de violencia aumentaron, otra vez, la percepción de inseguridad en la población carioca ${ }^{4}$. Arrastões ${ }^{5}$, asaltos a mano armada, secuestros, balaceras, balas perdidas, masacres y rebeliones en las cárceles" (Pereira Leite, 2012, p. 379) se volvieron parte de la cotidianidad de Río de Janeiro.

Los arrastões y el aumento de asaltos a mano armada ampliaron la inseguridad de los ciudadanos en el espacio público; los secuestros representaron, para las clases altas, la imprevisibilidad con la cual la violencia puede golpear, incluso en el ámbito 
$6 \quad$ La "ciudad formal" es toda aquella parte de la ciudad que no está compuesta por favelas. En Río de Janeiro, la "ciudad formal" es llamada también "asfalto", en contraposición a las favelas, que, en un comienzo, no tenían calles asfaltadas.

7 Por ejemplo, en el 2009 en toda la ciudad murieron ocho personas por balas perdidas, o sea el " $0,2 \%$ del total de personas que murieron por armas de fuego" (Penglase, 2011, p. 413).

8 La zona central de la ciudad, donde se encuentran todos los edificios del Gobierno Federal.

$9 \quad$ La zona de la ciudad donde está situado el estadio Maracaná

10 En Río de Janeiro las favelas están situadas, principalmente, en las colinas.

11 Uno de los barrios más ricos de la ciudad.

12 Conjunto de favelas situadas fuera de la parte central y más valorada económicamente de la ciudad.

\footnotetext{
13 Una de las maneras en las que viene
} comúnmente denominada Río de Janeiro. doméstico. Las rebeldías en las cárceles muestran el nivel de desesperación alcanzado por parte de un grupo de individuos pertenecientes a las clases sociales más pobres, evidenciando de esta manera que la existencia de un sistema social fuertemente desigual representaba una amenaza para las categorías socialmente incluidas. Las masacres de civiles inermes llevadas a cabo por parte de miembros de la policía, que en esos años se volvieron tristemente numerosos (Ramos \& Paiva, 2007), mostraron a la ciudad que también los representantes del Estado se estaban manchando de crímenes crueles, eliminando la esperanza de que representan la solución al clima de violencia que se creó en Río de Janeiro. En fin, la posibilidad de ser alcanzado por una bala perdida "símbolo de la violencia imprevisible, incontrolable y excesiva" (Penglase, 2011, p. 412) provocó en los residentes de toda la ciudad un gran sentimiento de angustia.

Una de las peculiaridades de Río de Janeiro es la cercanía entre "la ciudad formal" ${ }^{y}$ las favelas. Por este motivo, las guerras entre bandas criminales dentro de los barrios pobres pueden provocar víctimas también entre la población más rica. Comparado con los niveles de victimización de los residentes de las favelas, el riesgo de que los residentes del "asfalto" sean alcanzados por balas perdidas es muy bajo ${ }^{7}$. Sin embargo, una encuesta reciente muestra que el $60 \%$ de los residentes de la ciudad tiene miedo de ser alcanzado por una bala perdida (Monteiro, 2013). Además, los medios de comunicación reservan mucho espacio a las noticias de personas golpeadas por una bala perdida afuera de las favelas, aumentando la percepción de inseguridad de este sector de la población (Lannes Fernandes, 2013).

Si es verdad que al aumentar la visibilidad de la violencia en una determinada ciudad aumenta también la percepción de que esta sea violenta, es también verdad que la visibilidad de la violencia depende de quienes son sus víctimas. Por ejemplo, aunque en América Latina es mayor la violencia dentro de los territorios de la pobreza urbana (Briceño-León, 2005), la misma posee una visibilidad mucho menor de la que victimiza las categorías sociales más ricas. Las palabras de Mariano Beltrame, Secretario de Seguridad de Río de Janeiro del 2007 al 2016, confirman el hecho que diferentes niveles de inclusión en la esfera de los derechos de ciudadanía corresponden a diferentes niveles de visibilidad de la violencia.

La configuración geográfica de la ciudad es una característica peculiar que se refleja en el modelo de criminalidad que se verifica en Río de Janeiro. La violencia aquí no es periférica como, por ejemplo, en Brasilia. Esta tiene a Ceilandia, que es una ciudad satélite y es una región con un elevado índice de violencia, pero que está localizada a $40 \mathrm{~km}$ del Plan Piloto ${ }^{8}$. Eso reduce la percepción de inseguridad de los residentes del Distrito Federal.

Aquí [en cambio], en Tijuca', en la Zona Norte [de Río de Janeiro], nosotros tenemos diecinueve favelas y el ciudadano de la clase media está ahí abajo ${ }^{10}$ que ve la televisión mientras su hijo va a la escuela. Tenemos ocho favelas alrededor de Copacabana ${ }^{11}$, es ahí donde se instala la violencia. Aquí la violencia no es periférica, es visible. Y los grupos criminales que están en esos lugares bajan hacia el asfalto y afrontan el ciudadano. (...) Un disparo en Copacabana es una cosa, en el Alemão ${ }^{12}$ es otra. Una acción de la policía en Copacabana, tiene una repercusión muy grande porque las bandas criminales [presentes en las favelas cercanas] están a pocos metros de las ventanas de la clase media (Beltrame, 2009, p. 80).

Se ve cómo la violencia de Río de Janeiro es visible, dado que es central y localizada en las cercanías de los barrios de las clases medias y altas: "un disparo en Copacabana es una cosa, en el Alemão es otra". Es interesante notar cómo el Secretario de Seguridad no se incomoda por esconder el hecho que al generar noticia y, en consecuencia, al tener un peso político, sea únicamente válida la violencia a la cual están expuestos los residentes de los barrios de las clases medias y altas. Los únicos que, en su texto, Beltrame (2009) llama "ciudadanos".

Ha sido demostrado cómo "violencia y tráfico de drogas son elementos que hoy configuran la imagen de la Ciudad Maravillosa"13 (Pereira Leite, 2008, p. 115). De esta manera, la ciudad se ha vuelto "un caso ejemplar, casi paradigmático, de metrópolis afectada por la (in) seguridad pública" (Machado da Silva, Pereira Leite \& Fridman, 2005 , p. 4). Tal representación de Río de Janeiro como caso paradigmático de ciudad violenta va más allá del contexto nacional brasileño. Monteiro (2013) destaca que, desde los años de 1990, el alto índice de homicidios de Río de Janeiro la hizo identificarse, a nivel mundial, como una ciudad famosa por ser violenta. De la misma idea son Huguet 
14 En Brasil, las políticas de seguridad y el manejo de las fuerzas de policía están bajo el control de los Estados y no del Gobierno Federal o Municipal.

$15 \quad$ En Brasil, las muertes causadas por agentes de policía en servicio vienen llamadas "actos de resistencia", nombre que indica la inmediata absolución de los agentes del Estado los cuales, se supone apriorísticamente, matarían únicamente para resistir a eventuales ataques contra sus personas. y Szabó de Carvalho (2008), quienes recuerdan que Río es conocida por ser una de las ciudades más bellas del planeta, pero también una de las más violentas.

La fama de una ciudad influye, junto al alcance efectivo de la violencia, en permitir que un determinado espacio pase a ser percibido como violento fuera de las fronteras nacionales. Por esta razón, aunque en el 2013 Río de Janeiro contaba con una tasa de 19 homicidios cada 100.000 habitantes, era más conocida a nivel mundial por ser más violenta que la "desconocida" ciudad brasileña João Pessoa, que en el mismo año detenía una tasa de 79 homicidios cada 100.000 habitantes, la más elevada del país. Así, para ser percibida como violenta, la visibilidad internacional de la violencia de una ciudad puede tener un peso mayor que el real andamiento de la misma. En tal perspectiva, por ejemplo, la fama internacional de la película Ciudad de Dios, que narra los conflictos armados entre los grupos criminales de una favela carioca, puede, efectivamente, tener una influencia mayor que la tasa de homicidios.

Lo anteriormente descrito es sostenido también por Wilding (2010, p. 730), el cual afirma que el fenómeno de la violencia urbana pasa a ser asociado a Río de Janeiro porque la criminalidad violenta de esta ciudad "recibe una cobertura exagerada dentro de los medios de comunicación".

Es importante considerar también que Río de Janeiro es una ciudad turística. Por esta razón, si las personas que la visitan corren el riesgo de volverse víctimas de violencia, la ciudad tiene una posibilidad mayor de ser considerada como violenta a nivel internacional. Es este sentido, es importante notar que:

En Río de Janeiro, la tasa de asaltos llevados a cabo con violencia, o con la amenaza de la misma, que se registró por parte la policía, pasó de 263 cada 100.000 habitantes en el 1980 a 830 cada 100.000 habitantes en el 2009 (Misse, 2011, p. 13).

No sorprende, entonces, que, fuera de las fronteras nacionales, Río de Janeiro sea percibida como violenta también a causa de los robos que pueden padecer los turistas internacionales que cada año visitan la ciudad. Por ejemplo, en algunos sitios web de viajantes, Río de Janeiro aparece al doceavo (Smith, sin fecha) y sétimo (Okafor, 19 de marzo de 2015) lugar en la lista de las ciudades más violentas del mundo. En cambio, si se toma en consideración únicamente la tasa de homicidios, Río de Janeiro no aparece ni siquiera entre las primeras cincuenta ciudades más violentas del mundo (Macias \& Engel, 23 de enero de 2015).

\section{Estrategias de reducción de la visibilidad de la violencia urbana}

El proceso de pacificación de las favelas inició en diciembre del 2008. En aquel momento, el gobierno del Estado de Río de Janeiro ${ }^{14}$ pertenecía a Sergio Cabral y la Secretaría de Seguridad al ya mencionado Mariano Beltrame. Bajo el comando de estas figuras, las cuestiones relacionadas a la seguridad de la ciudad de Río de Janeiro fueron, en un primer momento, tratadas a través del uso masivo de la tradicional "metáfora de la guerra" que había sido activada por la mayoría de los gobiernos al poder de la ciudad y el Estado de Río de Janeiro en los últimos treinta años:

Río llegó a un punto en el que, infelizmente, se exigen sacrificios. Sólo que es difícil aceptarlo, pero para detener el poder de fuego de los bandidos, serán diezmadas vidas. (...) Es una guerra, en una guerra hay heridos y muertos (Entrevista a Beltrame, citada en Ribeiro, Dias \& Carvalho, 2008, pp. 10-11).

El resultado de tal abordaje al problema de la violencia fue un aumento de las mega operaciones de la policía dentro de las favelas y, en consecuencia, de las muertes causadas por los "actos de resistencia"15 (Machado da Silva, 2010 b; Misse, Cristoph Grillo, Teixeira \& Néri, 2013). En un primer momento, entonces, la intención de "llevar la paz" dentro las favelas, a finales del 2008, podría ser considerada como un cambio de rumbo y un distanciamiento de la estrategia y retórica beligerante actuadas hasta aquel momento por parte de la administración local. Pero, a través de un análisis en profundidad de los resultados obtenidos por el proceso de pacificación, se demuestra que las Unidades de Policía Pacificadora tienen, como principal objetivo, el de disminuir la visibilidad de la violencia en la ciudad. 
De hecho, estas procuran: a) eliminar el control territorial armado de las bandas criminales dentro de las favelas incluidas en el programa, o sea, las más visibles de la ciudad a causa de su cercanía con los barrios de las clases medias y altas; y b) en consecuencia, disminuir las muertes provocadas por las guerras territoriales entre bandas rivales y las incursiones violentas de la policía en estos territorios. En otras palabras, el objetivo oficial de las UPPs coincide con la disminución de aquellas formas de violencia que más han influenciado, tanto a nivel local como internacional, en la creación de la imagen de Río de Janeiro como "ciudad violenta".

Como mostraré en las próximas páginas, en los años sucesivos a la implementación del proceso de pacificación de las favelas, la ciudad vivió una disminución de los índices de criminalidad, particularmente en relación a los homicidios. Aunque las autoridades locales han dado los méritos de tal reducción a la creación de la UPPs, en realidad estas no tienen toda la responsabilidad de lo sucedido.

Además de la reducción de los homicidios, en el mismo periodo, se verificó también una reducción de los robos callejeros en la ciudad, sobre todo en los barrios de las clases medias y altas posicionados en los entornos de las favelas pacificadas (Cano, Borges \& Ribeiro, 2012). Las UPPs tienen la tarea de controlar únicamente las favelas incluidas en el proceso de pacificación. No obstante, sus miembros, con el objetivo de reducir el índice de robos, patrullan también las zonas circundantes (Saborio, 2017).

La introducción del Sistema Integrado de Metas (SIM) por parte de la Secretaría de Seguridad jugó un papel decisivo en favorecer el control del "asfalto" por parte de los miembros de las UPPs. El objetivo oficial del SIM fue colmar la falta de integración entre las diferentes fuerzas de policía, elemento que Macaulay (2012) señaló como uno de los principales factores que obstaculizan la prevención y represión de la criminalidad en Brasil. Con tal objetivo, el SIM establece metas de reducción de determinados índices de criminalidad que, según la Policía Civil y la Policía Militar, tienen que alcanzar en áreas de jurisdicción común. Antes del SIM, las sedes tradicionales de la Policía Militar, las UPPs (que, formalmente, también hacen parte de la Policía Militar) y las sedes de la Policía Civil, perseguían objetivos separados de reducción de la criminalidad. Con la introducción del SIM, en el 2009, el territorio de la ciudad pasó a ser dividido en Áreas Integradas de Seguridad Pública (AISPs). Dentro de cada una de estas, las diferentes fuerzas de policía trabajan de manera conjunta con el objetivo de reducir algunas formas de criminalidad. En las AISPs que alcanzarán las metas, todos los miembros de la Policía Civil, la Policía Militar y de las UPPs, obtenían un premio monetario. Estas se calculan cada seis meses y, a los policías de las AISPs que las alcanzaran, se les añadía el premio al salario base. En la AISP que alcanza el primer lugar, cada policía recibe una paga extra de $R \$ 9.000$, en la segunda de $R \$ 6.000$, en la tercera $R \$$ 4.500 , mientras que, en todas las demás que alcanzan las metas, cada policía recibe un premio de $\mathrm{R} \$ 3.000$ (Ganem Misse, 2014, p. 687).

Las metas se dan por los siguientes crímenes, en orden de importancia: letalidad violenta ${ }^{16}$, robos de automóviles y robos a transeúntes en la vía pública. 0 sea, las metas regulan únicamente los crímenes que más suscitan preocupación entre la población de Río de Janeiro (Monteiro, 2013) y, por ende, gozan de mayor visibilidad dentro de la opinión pública a nivel local. Al mismo tiempo, hay que tomar en consideración el hecho de que, como mencioné en la sección precedente, los robos callejeros aumentan la percepción de inseguridad entre los turistas extranjeros que, cada año, visitan Río de Janeiro. EI SIM, entonces, puede ser entendido como una estrategia de reducción de aquellos crímenes que gozan de una mayor exposición también entre la opinión pública internacional.

\section{Reducción de la violencia visible y producción del consenso}

No cabe duda de que las UPPs consiguieron reducir drásticamente la presencia de criminales visiblemente armados dentro de las favelas que fueron incluidas en el proceso de pacificación. El debate se vuelve más complejo cuando se analiza la reducción del número de homicidios que se verificó en la ciudad después de la implementación de la UPPs. Según las estadísticas oficiales, en el periodo que va del principio del proceso 
de pacificación, a finales del 2008, hasta el 2011, se verificó una reducción del $75 \%$ de los homicidios en las favelas pacificadas, pasando de 14 a 5 episodios al mes (Cano, Borges \& Ribeiro, 2012).

Datos más recientes nos muestran que, del 2007 al 2014, en las favelas pacificadas se pasó de una tasa de homicidios del 30,9 a una del 7,4 cada 100.000 habitantes (ISP, 2015). Estos resultados permitieron que las autoridades locales presentaran el proceso de pacificación como un éxito y un avance en los derechos de las poblaciones marginalizadas de la ciudad. Por otra parte, Ganem Misse (2014) demuestra cómo la reducción de los homicidios no está limitada a los territorios pacificados, sino que incluye todo el Estado de Río de Janeiro.

En el 2008, antes de la implementación de las UPPs, la tasa de homicidios de la ciudad de Río de Janeiro era de 38 por cada 100.000 habitantes, mientras que en el 2012 la ciudad registró la tasa de homicidios más baja de los últimos veinte años: 19 cada 100.000 habitantes. Para los representantes del gobierno y de la Policía Militar, tal reducción es resultado directo de la política de pacificación de las favelas (Monteiro, 2013; ISP, 2015). Sin embargo, la tasa de homicidios presentaba una gradual disminución desde el 2000 (Cano, Borges \& Ribeiro, 2012; Ganem Misse, 2014), o sea, mucho antes de la llegada de las UPPs en las favelas. Esto significa que la correlación entre las UPPs y la reducción de los homicidios, a nivel citadino, parece ser menos directa respecto a lo afirmado por parte de las instituciones locales. Además, en el 2010 se verificó una ulterior reducción de la tasa de homicidios en todo el Estado de Río de Janeiro y no sólo en las favelas pacificadas. Por esta razón, para Ganem Misse la reducción de los homicidios tiene que ser atribuida, principalmente, al SIM y no a las UPPs. De hecho, el SIM fue implementado en todo el Estado de Río de Janeiro, permitiendo que se disminuyeran los homicidios en todo su territorio, mientras que las UPPs fueron posicionadas únicamente dentro de la ciudad de Río de Janeiro, capital del Estado. En otras palabras, más que la intención de "llevar la paz" dentro las favelas, fueron los premios monetarios dados a los policías los capaces de influir en la reducción de los homicidios dentro de cada AISP.

Conjuntamente, hay que tomar en consideración que, para las autoridades locales, el resultado más importante de las UPPs fue el de reducir el $85 \%$ de los "actos de resistencia" dentro de las favelas pacificadas, los cuales pasaron de 136 en el 2008 a "solo" 20 en el 2014 (ISP, 2015). En relación a este punto, Ganem Misse (2014) hace notar que las UPPs resultaron ser, en primer lugar, una política de protección de la población marginalizada contra la violencia de la misma policía. Además, desde el 2010, año en el que los "actos de resistencia" fueron incluidos dentro de las metas del SIM, estos tuvieron una fuerte disminución en todo el Estado y no sólo en las favelas pacificadas. Entonces, para los "actos de resistencia", así como para los homicidios, la reducción parece ser responsabilidad también del SIM y no únicamente de las UPPs. En otras palabras, los policías se mostraron "más eficaces" en reducir los homicidios y en asesinar menos, sólo después de que pasaron a recibir un premio monetario para que así fuese. Este último elemento parece ser la motivación que llevó a las autoridades a dar menos relevancia, en el debate público, al SIM respecto a las UPPs en la reducción del crimen violento que se llevó a cabo dentro de la ciudad.

Aunque no existe un acuerdo sobre la causalidad de la disminución de los homicidios y los "actos de resistencia", las autoridades, apoyadas por los medios de comunicación, demostraron, casi de forma unánime, un "entusiasmo acrítico" (Machado da Silva, 2010a) en relación a las UPPs. Por lo tanto, el proceso de pacificación pasó, en un primer momento, a ser aclamado por los residentes de la "ciudad formal" como la nueva

$17 \quad$ En el 2013 aumentó el número de conflictos armados dentro de las favelas pacificadas entre miembros de bandas criminales y agentes de las UPPs, lo que llevó a un nuevo aumento de la visibilidad de la violencia (Saborio, 2017). solución al problema de la criminalidad (Fridman, 2014), en particular en relación a las formas de violencia más visibles. Desde el comienzo de la pacificación, hasta el $2013^{17}$, se verificó un acuerdo tácito entre la opinión pública y las autoridades, en el cual la reducción de la tasa de homicidios justificaba un modelo de policía represiva dentro de las favelas pacificadas (Machado da Silva, 2015; Freire, 2014).

Además del factor "homicidios", las UPPs conquistaron la aprobación de la opinión pública al mostrarse como una política de seguridad realizada bajo una óptica de "defensa del espacio" y neutralización de las poblaciones socialmente excluidas (Saborio, 2014; Saborio, 2017). Las UPPs consiguieron proteger las zonas más valorizadas de la ciudad 
reduciendo los robos y los conflictos armados en las favelas cercanas. Desde los años 1980, las categorías socialmente incluidas aceptaron políticas de seguridad pública abiertamente hostiles a la población pobre de la ciudad y que se usaban la "metáfora de la guerra" (Saborio, 2016). Igualmente, la misma población aceptó la militarización permanente que, gracias a las UPPs, se llevaba ahora a cabo con una retórica basada en el concepto de "paz" (Saborio, 2014). No sorprende, entonces, que el coronel Robson Rodrigues (2015, p. 10), ex comandante de todas las UPPs, afirmara que el proceso de pacificación dio a la Policía Militar de Río de Janeiro "una legitimidad que nunca había obtenido". O sea, la legitimidad proveniente de los que Beltrame, el ex Secretario de Seguridad, llama "ciudadanos" en la segunda parte de este artículo: las clases medias y altas, en particular las personas que residen en las zonas más valorizadas y visibles mediáticamente de la ciudad.

Lo dicho muestra cómo uno de los objetivos principales, aunque no oficial, de las UPPs es el de proteger las zonas de la ciudad donde vive la población más rica y donde se llevaron a cabo los mega eventos deportivos. En su defensa, las autoridades tienden a recordar que no todas las favelas pacificadas se ubican en tales zonas de la ciudad, pues las UPPs del Complexo do Alemão, Cidade de Deus, Batam, Villa Kennedy e Manguerinha están afuera del llamado "cinturón de seguridad" de los mega eventos. Sin embargo, un análisis más atento nos muestra que existe una conexión significativa entre estas UPPs: todas fueron implementadas para responder a una emergencia provocada por hechos de violencia altamente visibles y como respuesta inmediata a la opinión pública.

Entre las UPPs apenas mencionadas, el caso de Cidade de Deus y Batam tienen características diferentes. Así como sucedió con Santa Marta, la primera favela en ser pacificada, Cidade de Deus y Batam, donde fueron implementadas respectivamente la segunda y la tercera UPPs, eran ya ocupadas antes que se inaugurase oficialmente el proceso de pacificación y las fuerzas de policía que ocupaban las favelas pasasen a ser denominadas Unidades de Policía Pacificadora. Willis y Prado (2014) explican que Cidade de Deus fue ocupada por parte de la Policía Militar como resultado de una ola de violencia que se dio en la favela y, en consecuencia, en los barrios cercanos de la "ciudad formal". La favela ingresó oficialmente a formar parte de la política de pacificación en febrero del 2009, poco después de su inauguración. De esta manera, las autoridades tomaron ventaja del hecho de que la favela ya estaba ocupada por parte de las fuerzas de policía. Además, "llevando la paz", se consiguió disminuir la imagen de Río de Janeiro como ciudad violenta, imagen que había aumentado internacionalmente a causa de la famosa película Ciudad de Dios, ambientada en esa favela.

Dos días después de la inauguración de la UPP de Cidade de Deus, fue el turno de Batam de volverse oficialmente una "favela pacificada". Aquí, como en Cidade de Deus, las fuerzas de la Policía Militar ocupaban el territorio desde antes del comienzo del proceso de pacificación. Al día de hoy, Batam es la única favela pacificada que no estaba precedentemente controlada por el tráfico ${ }^{18}$, sino por un grupo de milicianos. Las milicias son formadas por ex policías, bomberos y policías fuera de servicio, los cuales sustituyeron a las bandas del tráfico en parte de las favelas de la ciudad. Donde se establecen, las milicias pretenden ser pagadas por los residentes de las favelas a cambio de "protección" y, en un segundo momento, pasan a controlar la venta abusiva de gas de cocina, televisión por cable y el transporte público (Cano \& Looty, 2008). La favela Batam fue ocupada por parte de la Policía Militar luego de un evento trágico que provocó una conmoción en la opinión pública: los milicianos capturaron, torturaron y mataron un grupo de periodistas que se habían infiltrado en la favela para documentar la actividad ilícita de la organización criminal (Da Silva, 2011). En este caso, como en el de Cidade de Deus, las autoridades aprovecharon el hecho de que la favela estuviera ocupada por la Policía Militar para instalar en esta una UPP y lanzar un mensaje tranquilizador a la ciudad que estaba trastornada por la muerte de los periodistas.

La ocupación del Complexo do Alemão se llevó a cabo en el 2010. Antes de la implantación de las fuerzas de pacificación, este conjunto de favelas era el ex cuartel general del Comando Vermelho, la organización del tráfico más potente en Río de Janeiro. La pacificación del Complexo do Alemão fue la respuesta del gobierno a una serie de bloqueos de la vía pública realizados en la "ciudad formal" por miembros del 
Comando Vermelho, los cuales incendiaron buses como forma de protesta contra el avance del proceso de pacificación de las favelas. Muchas de las favelas pacificadas se encontraban precedentemente bajo el control de tal organización y, el ingreso estable de la policía en dichos territorios, le provocó ingentes pérdidas económicas (Willis \& Prado, 2014).

La inauguración de la UPP Mangueirinha, la única presente en el Estado de Río de Janeiro, pero afuera de la capital, se llevó a cabo después de la "masacre de Chatuba", la cual generó un gran clamor a nivel nacional (Miagusko, 2014). En la masacre, seis jóvenes fueron torturados y asesinados de manera brutal por parte de traficantes, los cuales confundieron a las víctimas por miembros de un grupo criminal rival. La preocupación que este episodio generó en la ciudadanía llevó a las autoridades a inaugurar la UPP Mangueirinha en la favela donde se perpetró la masacre.

En fin, la ocupación de la favela Vila Kennedy en el 2014 fue el resultado de una feroz guerra territorial entre grupos criminales opuestos que, por más de un mes, llenó las primeras páginas de los periódicos locales. Algo parecido pasó en el 2015, cuando las bandas criminales de las favelas Chapadão y Pedreira entraron en guerra. En esa ocasión, el Secretario de Seguridad de Río de Janeiro amenazó a las partes en conflicto que su continuación habría significado la implantación de una nueva UPP en esas localidades (Sabóia, 2015). El mensaje de las autoridades a los grupos criminales fue claro: a cada conflicto armado que aumente el nivel de visibilidad de la violencia en la ciudad, podría corresponder la implantación de una nueva UPP.

\section{Conclusiones}

En los años 1980 se consolidó la percepción, tanto a nivel local como internacional, de que Río de Janeiro fuese una ciudad violenta 0 , incluso, en guerra. Tal situación no se dio de un día para otro, sino que fue el resultado de un progresivo aumento, que comenzó en los años 1950, de formas de violencia altamente visibles. Como se observó, la visibilidad de la violencia urbana depende de varios factores, entre los cuales destacan los tipos de personas que son víctimas y en qué parte de las ciudades se lleva a cabo. En el caso específico de Río de Janeiro, la visibilidad de la violencia está vinculada a la cercanía entre las favelas y los barrios de las clases medias y altas.

Las Unidades de Policía Pacificadora han sido presentadas por parte de las autoridades como la solución al problema de la violencia imperante dentro de las favelas de la ciudad. Sin embargo, el artículo muestra que estas tienen, como objetivo principal, disminuir el nivel de visibilidad de la violencia. De hecho, las UPPs han sido implementadas, casi exclusivamente, en las cercanías de las áreas más valorizadas de Río de Janeiro, las cuales, recientemente, hospedaron los dos mayores eventos deportivos a nivel mundial: el mundial de fútbol en el 2014 y las Olimpiadas en el 2016.

Las UPPs consiguieron reducir drásticamente la presencia visible de bandas criminales armadas en las favelas pacificadas de la ciudad y las consecuentes guerras territoriales e incursiones violentas de la policía, las cuales causaban un elevado número de muertes. Sin embargo, en el artículo se evidencia el hecho de que las UPPs no son las únicas responsables de la reducción del índice de violencia que se verificó en la ciudad desde el 2008. El Sistema Integrado de Metas tuvo una función importante en la reducción de los robos y de la letalidad violenta provocada por civiles y miembros de la policía. Además, el premio monetario ofrecido a los policías incentivó a los agentes de las UPPs a patrullar también afuera de las favelas pacificadas, con el objetivo de reducir los robos en los barrios de las clases medias y altas.

Las UPPs no han sido implementadas únicamente en las favelas posicionadas cerca de las áreas más valorizadas de la ciudad. En algunas ocasiones estas han sido instaladas en favelas más lejanas, pero que, a causa de eventos violentos, vivían una situación de emergencia que momentáneamente atraía la atención de los medios de comunicación, aumentando la visibilidad de la violencia en toda la ciudad. 


\section{Referencias Bibliográficas}

Beltrame, J. M. (2009). Estratégias, política e gestão em segurnça no Rio de Janiero: um desafio de todos os cidadãos. En D. M. Zouain, F. B. De Oliveira, M. A. Ruediger, \& V. Riccio (Eds.), Desafios da gestão pública de segurança. Rio de Janeiro: FGV.

Briceño-León, R. (2005). Violencia, Sociedad y Justicia en América Latina. CLASCO. Recuperado de http://biblioteca.clacso.edu.ar/clacso/gt/20101109031803/briceno.pdf

Cano, I., \& Looty, C. (2008). Seis por meia dúzia? Um estudo exploratório do fenômeno das chamadas milícias no Rio de Janeiro. En Laboratório de Análise da Violência (LAV-UERJ) (Ed.), Segurança, tráfico e milícias no Rio de Janeiro (pp.. 48-83). Rio de Janeiro: Fundação Heinrich.

Cano, I., Borges, D. \& Ribeiro, E. (2012). Os donos do morro. Uma avaliação exploratória do impacto das Unidades de Polícia Pacificadora (UPP) no Rio de Janeiro. Río de Janeiro: Fórum Brasileiro de Segurança Pública, LAV/UERJ.

Cano, I., \& Riberio, E. (2014). A Seletividade das "Políticas de Pacificação" no Rio de Janeiro. En Moulin A., Penalva E \& Sant'Anna M. J (Eds.). Transformações Territoriais no Rio de Janeiro do Século XXI,123-156. Río de Janeiro: Gramma Editora.

Da Silva, F. M. (2011). Unidade de Policiamento Pacificadora - UPP: um processo de democratização dos espaços favelados no Rio de Janeiro?. Presentado al Seminario Urbanismo na Bahia urbBA, Salvador de Bahia: Universidade Federal da Bahia.

Freire, J. (2014). 'Violência urbana' e 'cidadania' na cidade do Rio de Janeiro. Tensões e disputas em torno das 'justas atribuições' do Estado. DILEMAS: Revista de Estudios de Conflictos e Controle Social, 7(1), 73-94.

Fridman, L. C. (2014). Delegação de poder discricionário. 0 sonho de paz. DILEMAS: Revista de Estudos de Conflito e Controle Social, 7(4), 611-623.

Ganem Misse, D. (2014). Cinco anos de UPP: Um breve balanço. DILEMAS: Revista de Estudos de Conflito e Controle Social, 7(3), 675-700.

Huguet, C., \& Szabó de Carvalho, I. (2008). Violence in the Brazilian favelas and the role of the police. New directions for youth development, 119, 93-109.

Instituto de Segurança Pública (ISP). 2015. Balanço de Indicadores da Política de Pacificação (2007-2014). Río de Janeiro: Secretaria de Segurança, ISP.

Lannes Fernandes, F. (2013). Youth gang members in Rio de Janeiro. The face of a 'Lost Generation' in an age of fear and mistrust. Bulletin of Latin American Research, 32(2), 210-223.

Macaulay, F. (2012). Deepening the Federative Pact? The Dilma government's approach to crime, justice and policing. Critical Sociology, 1-12.

Machado da Silva, L. A. (2010a). Afinal, qual è a das UPPs? Observatório das Metrópoles. Recuperado de http://www.observatoriodasmetropoles.ufrj.br/artigo_ machado_UPPs.pdf

Machado da Silva, L. A. (2010b). Violência urbana, segurança pública e favelas - 0 caso do Rio de Janeiro atual. Caderno CRH, 23(59), 283-300.

Machado da Silva, L. A. (2015). A experiência das UPPs. Uma tomada de posição. DILEMAS: Revista de Estudos de Conflito e Controle Social, 8(1), 7-24.

Machado da Silva, L. A., Pereira Leite, M., \& Fridman, L. C. (2005). Matar, morrer, civilizar. 0 problema da segurança pública. MAPAS: Monitoramento Ativo da Participação da Sociedade. Recuperado de http://www.ipardes.gov.br/pdf/cursos_ eventos/governanca_2006/gover_2006_01_matar_morrer_civilizar_luiz_silva.pdf

Macias, A. \& Engel P. (23 de enero de 2015). The 50 most violent cities in the world. Recuperado de http://www.businessinsider.com/the-50-most-violent-cities-inthe-world-2015-1\#50-cuernavaca-mexico-had-2545-homicides-per-100000residents-1 
Miagusko, E. (2014). UPP na Baixada: circulação, violência e mercado político nas margens. Presentato al 38. ${ }^{\circ}$ Encontro Anual da ANPOCS - Sobre periferias: novos conflitos no espaço público, Mina Gerais: ANPOCS.

Misse, M. (2008). Sobre a acumulação social da violência no Rio de Janeiro. CivitasRevista de Ciências Sociais, 8(3), 371-385.

Misse, M., Cristoph Grillo, C., Teixeira, C. P., \& Néri, N. E. (2013). Quando a polícia mata: Homicídios por «autos de resistência» no Rio de Janeiro (2001-2011). Rio de Janeiro: Booklink.

Misse, M. (2011). Crime e violência no Brasil contemporâneo: estudos de sociologia do crime e da violência urbana $\left(2^{\circ}\right.$ ed.). Rio de Janeiro: Lumen Juris.

Monteiro, J. (2013). Os efeitos da Política de Pacificação sobre os confrontos entre facções de drogas no Rio de Janeiro. Rio de Janeiro: FGV/IBRE.

Okafor, O. (19 de marzo de 2015). The 15 most dangerous cities in the world are. Recuperado de http://pulse.ng/world/danger-alert-the-15-most-dangerous-citiesin-the-world-are-id3583275.html

Penglase, R. B. (2011). Lost bullets: fetishes of urban violence in Rio de Janeiro. Anthropological quarterly, 84(2), 411-438.

Penglase, R. B. (2014). Living with insecurity in a Brazilian favela: urban violence and daily life. New Jersey: Rutgers University Press.

Pereira Leite, M. (2008). Violência, risco e sociabilidade nas margens da cidade: percepções e formas de ação de moradores de favelas cariocas. En Machado da Silva, L.A. (Ed.), Vida sob cerco: violência e rotina nas favelas do Rio de Janeiro (pp. 115-141). Rio de Janeiro: Nova Frontiera.

Pereira Leite, M. (2012). Da «metáfora da guerra» ao projeto de «pacificação»: favelas e políticas de segurança pública no Rio de Janeiro. Revista Brasileira de Segurança Pública|São Paulo, 6(2), 374-389.

Ramos, S., \& Lemgruber, J. (2004). Urban violence, public safety policies and responses from civil society. Recuperado de http://www.ucamcesec.com.br/wordpress/wpcontent/uploads/2011/06/SocialWatch04_BR_violence1.pdf

Ramos, S., \& Paiva, A. (ed). (2007). Mídia e Violência: novas tendências na cobertura de criminalidade e segurança no Brasil. Rio de Janeiro: CESeC.

Ribeiro, C., Dias, R., \& Carvalho, S. (2008). Discursos e práticas na construção de uma política de segurança: 0 caso do governo Sérgio Cabral Filho (2007-2008). En Laboratório de Análise da Violência (LAV-UERJ) (Ed.), Segurança, tráfico e milícias no Rio de Janeiro (pp. 6-16). Rio de Janeiro: Fundação Heinrich Böll no Brasil.

Rodrigues da Silva, R. (2015). Temos que reformular e modernizar a corporação. Cuadernos VIVARIO, 1(1), 12-17.

Sabóia, Gabriel, (3 de mayo de 2015). Na hierarquia da violência, Chapadão é o novo Alemão Recuperado de http://odia.ig.com.br/noticia/rio-de-janeiro/2015-05-03/nahierarquia-da-violencia-chapadao-e-o-novo-alemao.html

Saborio, S. (2013). The pacification of the favelas: Mega events, global competitiveness, and the neutralization of marginality. Socialist Studies/Études socialistes, 9(2), 130-145.

Saborio, S. (2014). The new military urbanism. Police repression and conflict in Rio de Janeiro. Etnografia e ricerca qualitativa, 7(3), 401-422.

Saborio, S. (2016). La territorializzazione dell'esclusione sociale e della violenza a Rio de Janeiro. Sicurezza e scienze sociali, 10, 180-189.

Saborio. S. (2017). Policía Pacificadora, legitimidad y prácticas de ocupación territorial. Antípoda. Revista de Antropología y Arqueología, 29, 105-122.

Smith, (sin fecha). 10 Most Dangerous Cities in The World to Travel. Recuperado de http://www.escapehere.com/destination/10-most-dangerous-cities-in-the-worldto-travel/2/ 
Wilding, P. (2010). «New Violence». Silencing women's experiences in the favelas of Brazil. Journal of Latin American Studies, 42(4), 719-747.

Willis, G. D., \& Prado, M. M. (2014). Process and pattern in institutional reforms. A case study of the Police Pacifying Units (UPPs) in Brazil. World Development, 64, 232-242. 